\title{
An assessment of the hydration status of recreational endurance athletes during mountain marathon events
}

\author{
Elizabeth Mahon $^{1}$, Allan Hackett ${ }^{1}$, Timothy Stott ${ }^{1}$, Keith George ${ }^{2}$, Ian Davies ${ }^{1}$ \\ ${ }^{1}$ Faculty of Education, Health and Community, Liverpool John Moores University, Liverpool, United Kingdom \\ ${ }^{2}$ Research Institute for Sport and Exercise Sciences, Liverpool John Moores University, Liverpool, United Kingdom \\ Email address: \\ E.Haywood@ljmu.ac.uk (E. Mahon)
}

\section{To cite this article:}

Elizabeth Mahon, Allan Hackett, Timothy Stott, Keith George, Ian Davies. An Assessment of the Hydration Status of Recreational Endurance Athletes During Mountain Marathon Events. American Journal of Sports Science. Vol. 2, No. 4, 2014, pp. 77-86. doi: 10.11648/j.ajss.20140204.12

\begin{abstract}
Changes in hydration status have been shown to have deleterious effects on physiological functioning, physical performance, mental decision making, and subjective sensation of effort. This exploratory study assessed the hydration status of athletes taking part in mountain marathon events and the implications on performance, cognition and well-being markers. Twenty-nine male recreational athletes from the Original Mountain Marathon (OMM) and nineteen from the Longmynd Hike $(\mathrm{LH})$ provided urine samples before, at mid-point (in the OMM) and after the events. Body mass; reaction time tests; and subjective ratings of well-being and effort were also collected/performed at these time points. Post-urine specific gravity $\left(\mathrm{U}_{\mathrm{sg}}\right)$ values [(OMM: 1.023 (range: $1.008-1.038) \mathrm{g} / \mathrm{ml}$; LH: 1.024 (range: $1.014-1.035$ ) g/ml] were significantly higher than pre-values [(OMM: 1.013 (range: $1.002-1.026) \mathrm{g} / \mathrm{ml}$; LH: 1.013 (range: $1.002-1.026) \mathrm{g} / \mathrm{ml}$ ] in both events $(p<0.01)$, but there was no significant change from mid- to post-event in the OMM $(p>0.05)$. There was no association between hydration status and overall performance in the $\mathrm{LH}$, whilst change in $\mathrm{U}_{\mathrm{sg}}$ from pre- to mid-event was positively associated with performance in the $\mathrm{OMM}(\mathrm{r}=.561, p=0.004)$. Whilst no associations were observed between hydration status and reaction time, rate of perceived exertion and subjective ratings of nausea showed positive associations with $\mathrm{U}_{\text {sg. }}$. These findings suggest that although changes in hydration status of this level may not significantly affect performance, they can impact on participant sensations of effort and well-being.
\end{abstract}

Keywords: Fluid, Exercise, Cognition, Running, Hill-Walking

\section{Introduction}

Prolonged exercise increases the requirement for water intake in order to maintain hydration. Even modest changes in hydration status (equivalent to $2 \%$ loss in body mass) can have deleterious effects on physical performance [1-2], mental decision making, physiological functioning and temperature regulation [3], all important safety considerations in a mountainous environment. Previous research has shown that during a two-day mountain marathon, only $13 \%$ of subjects finished well hydrated and $27 \%$ reported symptoms of dehydration such as headaches, cramps, and feeling faint [4]. Consuming fluid at regular intervals during exercise is therefore important to optimize performance and safeguard health and well-being.

Guidelines for fluid consumption during exercise have changed over the years; an ultra-distance runner in the 1940s
- 1960s was cited in Noakes (2003, p.199) [5]: “To run a complete marathon without any fluid replacement was regarded as the ultimate aim of most runners and a test of their fitness". In the 1970s, athletes were advised to stay ahead of thirst and maximising rehydration during events was of primary concern [6]. However, following several cases of hyponatremia as a result of over-hydration in endurance events [7], it has since been realised that taking on too much fluid can be as serious as taking on too little and therefore fluid replacement guidelines need to take into account a myriad of factors, such as: exercise intensity; duration; environmental conditions; and individual sweat rates. The International Olympic Committee (IOC) [8] state that the goal of drinking during exercise should be to limit dehydration to less than about $2 \%$ of body mass (BM), but 
not to drink so much that weight is gained during exercise.

Although it is commonly believed that dehydration equal to $2 \%$ BM loss can have a detrimental effect on endurance performance [9], the data regarding the effects in temperate conditions remains equivocal. Whilst several studies have observed detriments to performance [10-11], others have shown improvements in performance with markers of dehydration $[4,12]$. Therefore, more research is needed to resolve the impact of fluid balance on endurance performance. This is particularly important in prolonged exercise, as the longer the exercise duration the greater the cumulative effects of any differences between fluid needs and fluid replacement. As a result one would expect that dehydration or over-hydration in prolonged events would have the potential to have greater negative effects on performance and health.

The main objective of the present study was to assess the hydration status of athletes competing in mountain marathon events and to study the association between levels of hydration and performance. As a secondary objective, the associations between hydration status and cognitive performance and well-being were also investigated as these may be important to athletes whilst in mountain environments. The majority of research studying the effects of dehydration on cognitive function have used heat and exercise to induce rapid dehydration in laboratory settings [13], which may yield very different results to progressive and slower development of dehydration in temperate conditions out in the field. In addition, given the wide range of ages of the competitors that take part in mountain marathons, hydration status was compared with respect to age to determine whether, as published elsewhere [14-15]; older athletes are more prone to dehydration.

\section{Method}

\subsection{Study Design}

This cross-sectional study was designed to assess the hydration status of recreational athletes competing in two UK mountain marathon events in the autumn of 2009. Hydration status was then related to measures of overall performance, cognition, rate of perceived exertion (RPE) and subjective ratings of well-being.

\subsection{Setting}

The events used were the Original Mountain Marathon (OMM) and the Longmynd Hike (LH). The OMM is an unsupported (no food or drink is provided) two-day race combining fell-running and orienteering over mountainous terrain, held in a different region of the UK each year. The 2009 event was held at the end of October in the Elan Valley, Wales. Participants compete in teams of two and it is designed to test fitness and orienteering skills in extreme circumstances. Each team must carry all their equipment including that needed for an overnight camp. Participants are grouped into classes, dependent on fitness and experience:
Long (approximately $65 \mathrm{~km}$ over the two days), Medium (approximately $50 \mathrm{~km}$ ) or Short (approximately $45 \mathrm{~km}$ ).

The LH is an $80.5 \mathrm{~km}$ continuous, individual competitive mountain marathon set in the countryside of South Shropshire and the Welsh Marches UK, during the first weekend of October each year. The course includes approximately 2,400 $\mathrm{m}$ of climbing covering eight summits. No food is provided during the event; however water is available at the checkpoints on route.

\subsection{Participants}

Recreational athletes were targeted for entry into the study as a growing number of such athletes are taking up ultra-endurance racing [16]. Once ethical approval was granted for the study by the Liverpool (Adult) Research Ethics Committee, event organizers were approached via email for permission to recruit participants. Competitors were encouraged to participate through links on the event websites and flyers sent out with entry packs. In total 48 male participants were recruited to this study; sample characteristics were as follows: OMM $[n=29$; age (18-39 y: $n=16,40-49$ y: $n=7,50+\mathrm{y}: n=6$ ); class (long: $n=6$, medium: $n=12$, short: $n=11)]$, LH $[n=19$; age (18-39y: $n=$ 5, 40-49 y: $n=7,50+\mathrm{y}: n=7)$; course completion time $16( \pm$ 3) h]. There were various levels of participation in the study; this is reflected in the results.

\subsection{Protocol and Procedures}

All procedures described below were performed immediately prior to the start of the mountain marathons at the event headquarters, at the mid-point campsite at the end of day one (for the OMM only), and at the end of the events.

\subsubsection{Body Mass}

The BM of each participant was recorded using calibrated scales accurate to $0.1 \mathrm{~kg}$. Participants were weighed with their clothes on, but without shoes and accessories (caps, bottles, bags, wallets, keys etc). Total BM change was used as an indicator of fluid loss/gain.

\subsubsection{Estimation of Fluid Intake}

Fluid consumption was estimated by participants retrospectively (at mid-camp and post-event) to the nearest half-litre per hour by questionnaire [4].

\subsubsection{Urine Sampling and Analysis}

Urine specific gravity $\left(\mathrm{U}_{\mathrm{sg}}\right)$ was used to assess hydration status as the validity and reliability has been tested and it is considered one of the most appropriate methods for use in the field [17]. Percentage BM loss over the events was used as a second measure, however these data must be considered with caution as athletes were not weighed naked, and it is generally only considered accurate over relatively short time periods of 1 to 4 hours. When measurements are taken over intervals larger than this water exchange due to substrate oxidation and respiratory water loss become significant factors [18]. 
$\mathrm{U}_{\mathrm{sg}}$ was measured using a visual hand held refractometer (Model: Index Instruments Ref: 301). Liquid reference standards with an accuracy of \pm 0.0005 refractive index were used prior to each testing period to check for precision of the instrument. Participants were provided with a sample pot and were asked to provide a midstream urine sample as close as possible to the start, mid-point and the end of the events. $U_{s g}$ was assessed immediately on receipt of the sample in a well-lit area. During each testing period $10 \%$ of the samples were tested twice to test the reliability of the refractometer. Pre-event samples were considered euhydrated with values $\leq$ $1.020 \mathrm{~g} / \mathrm{ml}$. The ACSM's position on Exercise and Fluid Replacement (2007, p.385) [19] state that "a person with a first morning $U_{s g} \leq 1.020$ can be considered as euhydrated". Post-event samples were considered in the normal range with values between 1.013 and $1.029 \mathrm{~g} / \mathrm{ml}$; samples with values of $1.030 \mathrm{~g} / \mathrm{ml}$ or higher being considered to be significantly dehydrated; and values below $1.012 \mathrm{~g} / \mathrm{ml}$ were considered hyperhydrated. These values are based on what is typically seen during dehydration, exercise and rehydration [20].

\subsubsection{Cognitive Performance}

As mountain marathons involve competitors making quick navigational decisions, a choice reaction time test (CRT), considered a decision task, was the most suitable cognitive test to mimic the tasks involved in the 'real-life' setting. Diet and exercise interventions have shown that CRTs show greater sensitivity than simple reaction time tests [21]. A unique laptop-based CRT was designed, programmed and tested $(r=0.91)$ for this study. Participants completed a familiarisation trial prior to their first recorded attempt as prior testing (unpublished) showed it to be subject to learning effects which may affect the test-retest reliability.

\subsubsection{Subjective Measurements}

A questionnaire was designed to allow participants to rate their feelings of "thirst", "nausea", "tiredness", "physical fatigue" and "mental fatigue". These ratings were assessed by a 100-mm visual analogue rating scale labelled from "not at all" to "extremely". Participants were advised to mark a line through the scale at a point between the two extremes of the symptom being rated, which they considered to indicate the degree of the subjective feeling they were experiencing. The nature of these rating scales and their validity in relation to fluid and food consumption have been previously described [22], and have been used in studies of similar design [23].

To determine perception of fatigue during the event, participants were asked to provide a score using the Borg 15-point rating of perceived exertion (RPE) scale [24]. Ratings were taken at mid-point and at the end of the events. This scale has been adopted as the standard instrument for evaluating the perception of whole-body exertion during exercise and has been used by countless studies in the sports and exercise field in laboratory settings [21,25], in mountainous environments [26], and during ultra-endurance events [27].

Finally, mid- and post-event questionnaires were used to gather information on the occurrence of symptoms during the marathons that may be linked to dehydration (e.g. headaches), where fluid was obtained from during the events, and whether participants felt they had drank sufficient amounts.

\subsubsection{Overall Performance}

Overall performance was calculated as a reciprocal percentage of the winning time [4], times were age-adjusted and class-specific for the OMM:

200 - (individual time / winning time x 100)

\subsection{Statistical Procedures}

Statistical analyses were performed with SPSS (version 17.0). Descriptive statistics (mean, standard deviation (SD), minimum, maximum) were calculated for pre, mid and post $\mathrm{BM}$, percentage $\mathrm{BM}$ loss, $\mathrm{U}_{\mathrm{sg}}$ and cognitive test scores. The change in measurement from pre-, to mid-, to post-event was also calculated for these variables. Frequencies were reported for hourly estimates of fluid consumption, reported symptoms and number of participants meeting hydration cut-offs.

A one-way ANOVA was used to investigate differences in $\mathrm{U}_{\mathrm{sg}}$ values between participants of different age or class, and to determine whether there were any statistically significant differences in cognitive performance depending on hydration status. Paired sample $t$-tests were used to detect for significant differences between $\mathrm{BM}, \mathrm{U}_{\mathrm{sg}}$ and cognitive test scores at different time points. Independent sample $t$-tests were employed to identify any differences in $U_{\text {sg }}$ values between those reporting having experienced a symptom and those not. Pearson's and Spearman's rank correlation coefficients were performed to examine associations between $\mathrm{U}_{\mathrm{sg}}$ values and overall performance, and subjective ratings. All tests were two-tailed, with alpha $<0.05$.

\section{Results}

\subsection{Fluid Intake and Hydration Status}

Table 1. Hourly fluid consumption estimates

\begin{tabular}{llll}
\hline Fluid consumption & LH & OMM & \\
\hline$(\mathbf{L} / \mathbf{h})$ & $(\boldsymbol{n}=\mathbf{1 9})$ & Day 1 $(\boldsymbol{n}=\mathbf{2 9})$ & Day 2 $(\boldsymbol{n}=\mathbf{2 8})$ \\
\hline$<0.25$ & 5 & 10 & 9 \\
$0.25-0.5$ & 12 & 19 & 13 \\
$>0.5-1$ & 2 & 0 & 2 \\
$>1-1.5$ & 0 & 0 & 2 \\
$>1.5$ & 0 & 0 & 2 \\
\hline
\end{tabular}

Information from questionnaires revealed that the majority of participants in both events estimated their fluid consumption whilst running to be between $0.25-0.5$ L/hour (Table 1). In the $\mathrm{LH}$, water was available at the checkpoints on route; responses from questionnaires indicated that all runners made use of this. In addition, 74\% of participants carried their own drinks. In the OMM, no drinks were provided (with the exception of water at mid-camp) and so fluid was either obtained from that carried or from natural sources. On day one, $57 \%$ of participants carried their own drinks, whilst on day two this 
dropped to $32 \%$. A considerable percentage of both samples felt that they drank insufficient fluids: $37 \%$ of the $\mathrm{LH}$ and $64 \%$ of the OMM on day one and $52 \%$ on day two. Timing of intake and type of fluid consumed was not recorded.

Data regarding hydration status is presented in Tables 2 and $3 . \mathrm{U}_{\mathrm{sg}}$ significantly increased from pre- to mid-event (OMM: $t=-6.03, p<0.001, n=27$ ) and pre- to post-event (OMM: $t=-5.65, p<0.001, n=27$; LH: $t=-4.13, p=0.001$, $n=17)$. There was no further significant increase from midto post-event in the OMM. For both samples the mean pre-event $\mathrm{U}_{\mathrm{sg}}$ was considered euhydrated $(\leq 1.020 \mathrm{~g} / \mathrm{ml})$. Only two participants in each sample started the event with an $U_{\mathrm{sg}}>1.020 \mathrm{~g} / \mathrm{ml}$. In the $\mathrm{LH}$, the mean post-event $\mathrm{U}_{\mathrm{sg}}$ was $>$ $1.020 \mathrm{~g} / \mathrm{ml}$ but below that considered significantly dehydrated in sportsmen ( $>1.029 \mathrm{~g} / \mathrm{ml})$ [20]. Using this higher figure, just four participants in the LH sample were considered significantly dehydrated at the end of the event. In the OMM, only one participant was considered significantly dehydrated at the end of day one, but eight by the end of day two. There were no significant differences in $\mathrm{U}_{\mathrm{sg}}$ values between participants of different age or class.

BM significantly decreased from pre- to mid-event $(t=$ 5.83, $p<0.001, n=27$ ) and pre- to post-event (OMM: $t=$ 4.94, $p<0.001, n=27$; LH: $t=6.83, p<0.001, n=18$ ). There was no further significant decrease from mid- to post-event in the OMM. The mean percentage BM losses (LH: $1.4 \%$; OMM: $1.3 \%$ day one, $1.4 \%$ overall) were within the $0-2 \%$ range considered acceptable during endurance exercise [19]. In the $\mathrm{LH}$, four of the seventeen participants lost more than $2 \%$ of their BM during the event and no participants gained weight. In the OMM, three lost greater than $2 \%$ of their BM on day one and seven throughout the event as a whole. One individual lost more than $5 \%$ of their total BM. Conversely, two participants gained weight. However, there was no relationship between percentage BM loss and $\mathrm{U}_{\mathrm{sg}}$ in either sample.

In the LH there were no associations with $\mathrm{U}_{\mathrm{sg}}$ and overall performance in the event. In the OMM change in $U_{\text {sg }}$ from pre- to mid-event (day one) was positively associated with performance $(r=.561, p=0.004, n=24)$ (Fig.1).

Table 2. Hydration measures of OMM participants (**Significant difference in values between pre and post $(p<0.01)$, †t Significant difference in values between pre and mid $(p<0.01))$

\begin{tabular}{llll}
\hline \multirow{2}{*}{ Measurement } & Time point & \multicolumn{2}{l}{ OMM $(\boldsymbol{n}=\mathbf{2 7})$} \\
\cline { 3 - 4 } & & Mean (SD) & Range \\
\hline \multirow{2}{*}{$\begin{array}{l}\text { Specific gravity of } \\
\text { urine }\left(\mathrm{U}_{\mathrm{sg}}\right)(\mathrm{g} / \mathrm{ml})\end{array}$} & Mid & $1.013(0.006)$ & $1.002-1.026$ \\
& Post & $1.021(0.007) \dagger \dagger$ & $1.010-1.033$ \\
& Pre & $81.0(10.0)$ & $60.9-104.8$ \\
Body mass $(\mathrm{kg})$ & Mid & $80.0(10.2) \dagger \dagger$ & $60.3-104.5$ \\
& Post & $79.9(10.0)^{* *}$ & $60.7-103.0$ \\
& Day One & $1.3(0.7)$ & $-0.41-4.29$ \\
Body mass loss $(\%)$ & Overall & $1.4(1.5)$ & $-0.85-5.36$ \\
\hline
\end{tabular}

Table 3. Hydration measures of LH participants (**Significant difference in values between pre and post $(p<0.01))$

\begin{tabular}{llll}
\hline \multirow{2}{*}{ Measurement } & \multirow{2}{*}{ Time point } & \multicolumn{2}{l}{ LH $(\boldsymbol{n}=\mathbf{1 8})$} \\
\cline { 3 - 4 } & & Mean (SD) & Range \\
\hline Specific gravity of & Pre & $1.013(0.009)$ & $1.002-1.026$ \\
urine $\left(\mathrm{U}_{\mathrm{sg}}\right)(\mathrm{g} / \mathrm{ml})$ & Post & $1.024(0.007)^{* *}$ & $1.014-1.035$ \\
& Pre & $76.3(10.3)$ & $63.7-109.2$ \\
Body mass $(\mathrm{kg})$ & Post & $75.2(10.4)^{* *}$ & $63.0-108.7$ \\
Body mass loss $(\%)$ & Overall & $1.4(0.9)$ & $0.07-2.99$ \\
\hline
\end{tabular}

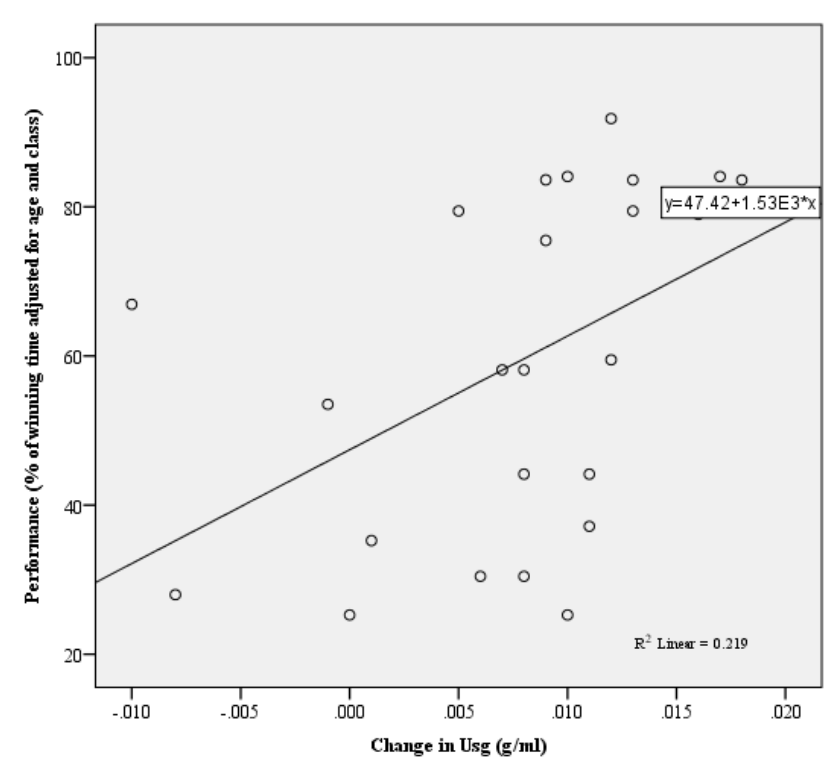

Figure 1. Change in Usg and performance during day one of the OMM

\subsection{Hydration Status and Cognitive Performance}

Reaction time tests were performed pre-, mid- and post-event to determine the effects of hydration status on cognitive function. The results are displayed in Table 4. In the $\mathrm{LH}$, mean time taken to respond to the reaction time test significantly increased from pre- to post-event. In contrast, there were no significant differences in reaction times between the three time points in the OMM. When grouped according to hydration status $(<1.013 \mathrm{~g} / \mathrm{ml} ; 1.013-1.029$ $\mathrm{g} / \mathrm{ml}$; and $>1.029 \mathrm{~g} / \mathrm{ml}$ ) there were no significant differences between groups in either absolute reaction times or changes in reaction times across the events (Fig.2).

Table 4. Choice reaction time test scores: pre, mid and post event $(* *$ Significant difference in values between pre and post $(p<0.01))$

\begin{tabular}{llll}
\hline \multirow{2}{*}{ Event } & Time & \multicolumn{2}{l}{ CRT (time taken to respond (ms)) } \\
\cline { 3 - 4 } & point & Mean (SD) & Range \\
\hline \multirow{2}{*}{ OMM $(n=25)$} & Pre & $3248(644)$ & $1970-4593$ \\
& Pid & $3314(624)$ & $2348-4840$ \\
LH $(n=17)$ & Pre & $3251(590)$ & $2461-4610$ \\
& Post & $3788(872)^{* *}$ & $2512-6328$ \\
\hline
\end{tabular}




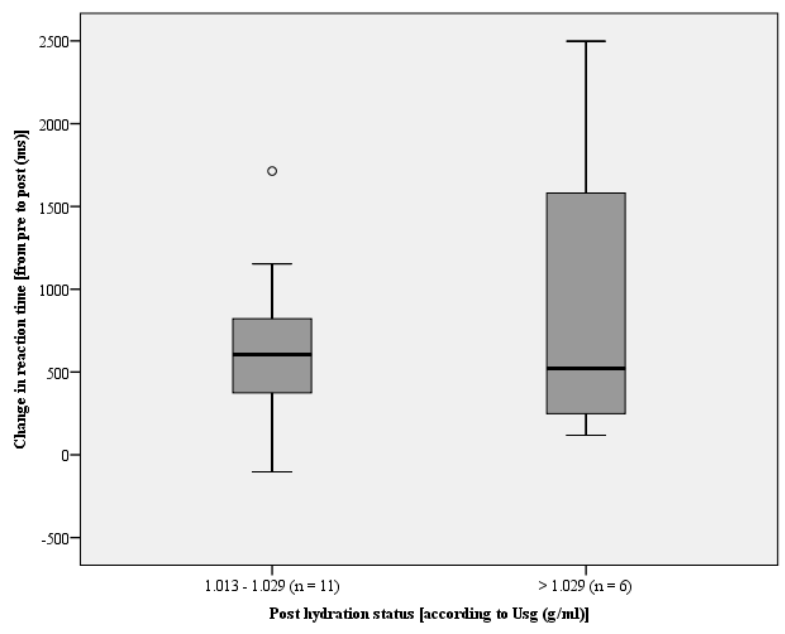

Figure 2. Change in choice reaction time with hydration status in LH participants ( Indicates outliers)

\subsection{Hydration Status and Subjective Measures of Well-Being}

Correlation analyses were performed to identify associations between hydration status (according to absolute, and changes in, $U_{\mathrm{sg}}$ values) and subjective sensations, such as those reported on the visual analogue scales and RPE scores. In both samples there were strong positive correlations between $\mathrm{U}_{\mathrm{sg}}$ values and self-reported ratings of nausea; in the $\mathrm{LH}$ post $\mathrm{U}_{\mathrm{sg}}$ was positively associated with ratings of nausea on the post subjective-measures questionnaire $(r=.534, p=.027, n=17)$, and in the OMM mid-event $\mathrm{U}_{\mathrm{sg}}$ values were positively associated with day one ratings of nausea $(r=.513, p=.007, n=26)$. In addition, in the OMM sample, mid-point RPE scores showed positive correlation with change in $\mathrm{U}_{\mathrm{sg}}$ values over day one $(r=.566$, $p=.005, n=23$ ).

On the mid- and post-event questionnaires participants were asked to report whether they suffered from any symptoms characteristic of dehydration. In the LH, mean post-event $U_{s g}$ values were higher in those suffering from headache, cramp, stomach discomfort and nausea, compared to those who did not report these symptoms. However, there were only significant differences in post $U_{\text {sg }}$ values between those experiencing headaches $[1.029( \pm 0.004) \mathrm{g} / \mathrm{ml} ; n=6]$ and those not $[1.022( \pm 0.008) \mathrm{g} / \mathrm{ml} ; n=11 ; p=0.044]$ (Fig.3) and those suffering from stomach discomfort $[1.031( \pm$ $0.004) \mathrm{g} / \mathrm{ml} ; n=4]$ and those not $[1.022( \pm 0.007) \mathrm{g} / \mathrm{ml} ; n=$ $13 ; p=0.025]$.

In the OMM, mean mid-event $U_{\text {sg }}$ values were significantly higher in those suffering from headaches $[1.029( \pm 0.003) \mathrm{g} / \mathrm{ml} ; n=4]$ than those not $[1.019( \pm 0.006)$ $\mathrm{g} / \mathrm{ml} ; n=22 ; p=0.007]$ (Fig.4); in those experiencing cramp $[1.025( \pm 0.005) \mathrm{g} / \mathrm{ml} ; n=12]$ than those not $[1.017( \pm 0.006)$ $\mathrm{g} / \mathrm{ml} ; n=14 ; p=0.001]$; in those who reported stomach discomfort $[1.027( \pm 0.004) \mathrm{g} / \mathrm{ml} ; n=6]$ compared to those who did not $[1.019( \pm 0.006) \mathrm{g} / \mathrm{ml} ; n=20 ; p=0.003]$; and in those who expressed having felt nauseous $[1.028( \pm 0.002)$ $\mathrm{g} / \mathrm{ml} ; n=7]$ in comparison to those who did not $[1.018( \pm$
0.005) $\mathrm{g} / \mathrm{ml} ; n=19$; $p<0.001]$, during day one. No significant differences were observed with post-event $U_{s g}$ values and day two symptoms.

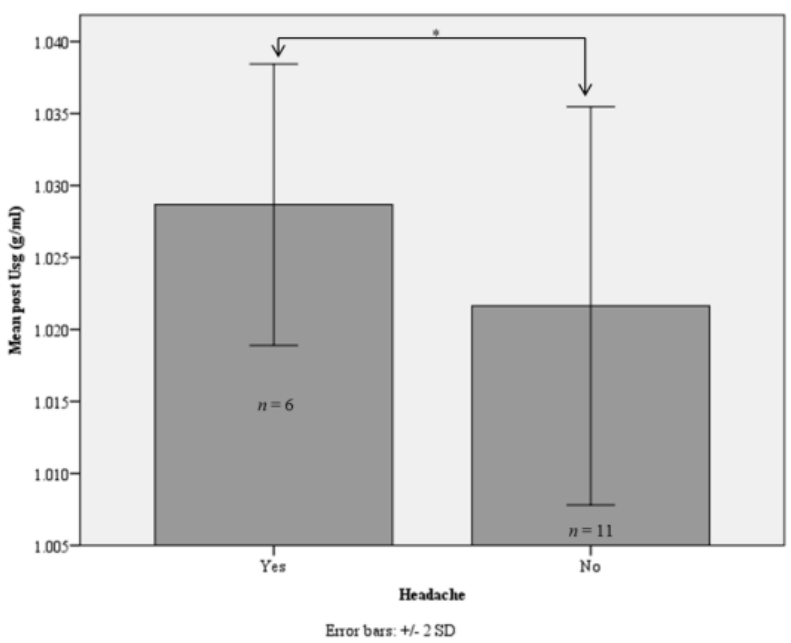

Figure 3. A comparison of post urine specific gravity and occurrence of headaches in LH participants (*Significant difference between groups ( $p<$ 0.05))

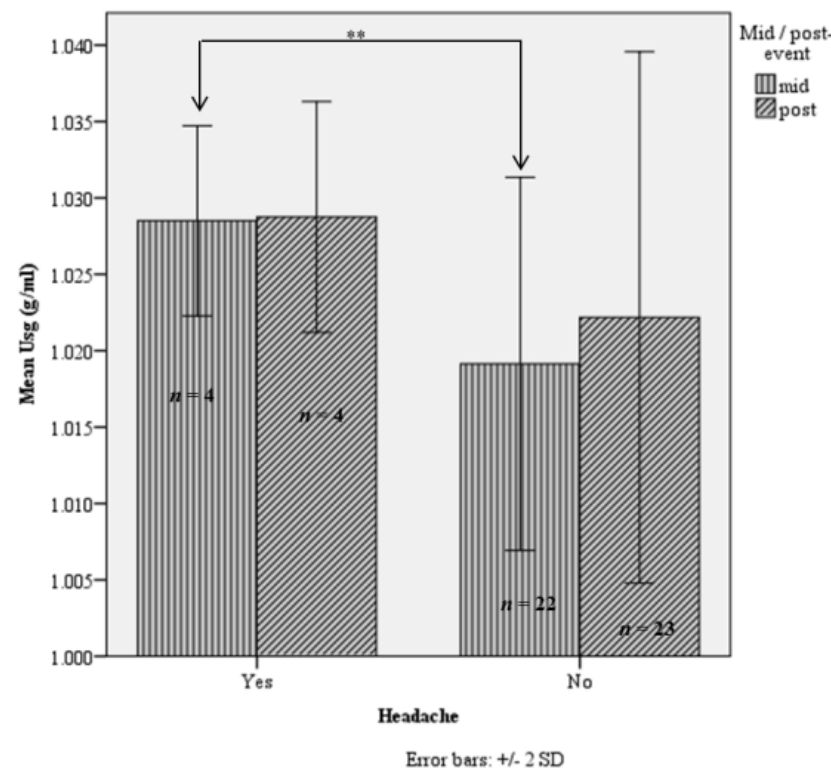

Figure 4. A comparison of urine specific gravity and occurrence of headaches mid and post-event in OMM participants (**Significant difference between groups $(p<0.01))$

\section{Discussion}

\subsection{Fluid Intake and Hydration Status}

Hourly fluid intake could not be determined exactly but estimates are similar to those in other mountain races. A study on mountain marathon athletes using the same method reported that over three-quarters of athletes estimated their fluid consumption to be $\leq 500 \mathrm{ml} / \mathrm{h}$ [4]. In studies where fluid intake was more precisely measured: intakes of 545 $\mathrm{ml} / \mathrm{h}$ have been published during a $44 \mathrm{~km}$ Swiss mountain marathon [28]; and $422 \mathrm{ml} / \mathrm{h}$ in runners during an $80 \mathrm{~km}$ 
mountain trail race [29]. Studies investigating different types of ultra-endurance events have found higher intakes when other disciplines apart from running are involved; intakes of $716 \mathrm{ml} / \mathrm{h}$ have been reported during an ironman triathlon [30]. The differences seen in fluid intake will not only be due to the format of the event, but also due to the environmental temperature and whether aid stations are available. In the present study, those competing in the OMM often had to rely on finding water from natural sources, whereas in the LH it was available at checkpoints. In those studies described above where fluid was measured, these values were all recorded from fluids taken from aid stations.

Due to different race conditions, comparing fluid intake is not the most useful measure, as requirements will differ, therefore it is more important to monitor the hydration status of athletes. In this study the specific gravity of urine samples was measured to assess hydration status. In the $\mathrm{LH}$, the post-event range in $\mathrm{U}_{\mathrm{sg}}$ values was $1.014-1.035 \mathrm{~g} / \mathrm{ml}$, representing wide variation in the hydration status of athletes completing the event (Table 3 ). Whilst the mean value (1.024 $\mathrm{g} / \mathrm{ml}$ ) was within the range considered normal $1.013-1.029$ $\mathrm{g} / \mathrm{ml}$ after exercise [20], four out of the 18 participants had values considered significantly dehydrated $(>1.029 \mathrm{~g} / \mathrm{ml})$. In the OMM, the mean $\mathrm{U}_{\mathrm{sg}}$ values were similar at the end of each day (Day 1 (mid): $1.021 \mathrm{~g} / \mathrm{ml}$; day 2 (post): $1.023 \mathrm{~g} / \mathrm{ml}$ ) (Table 2), although the range was larger at the end of day two and more participants were significantly dehydrated; eight post-event versus just one at mid-camp. This could suggest that some participants failed to sufficiently re-hydrate at mid-camp and started day two in an already dehydrated state. This agrees with findings from Clark et al. [4] who took a measure of hydration status at the start of day two across a range of mountain marathon events. They found that whilst some participants had managed to rehydrate over the mid-camp rest, others remained severely dehydrated. Conversely, four participants in the OMM had $\mathrm{U}_{\mathrm{sg}}$ values < $1.013 \mathrm{~g} / \mathrm{ml}$ at the end of both days, suggesting potential over-drinking, which is generally associated with inexperienced runners. In studies where athletes have had to qualify for entry into the mountain race, and thus include possibly more experienced runners, there have been no reports of athletes drinking to excess [29].

A surprising finding of the present study, given the wide range of ages represented, was that no significant differences were found in hydration status with age. Several studies have reported that older athletes are more likely to become dehydrated $[14,15]$. Whilst older adults have been shown to consume adequate volumes of fluid on a daily basis under normal conditions, an age-related blunting of thirst response to water deprivation or exercise exists, making them more susceptible to dehydration [31,32]. One suggestion for this is that older adults tend to have an increased resting plasma osmolality, which may lead to a higher osmotic operating point for thirst sensation [33]. As a result older adults are often slower to restore body fluid homeostasis. It is possible that in the present study there were not large enough numbers of older athletes to detect significant differences in hydration status.

The majority of field studies that have estimated hydration status have used percentage BM loss as an indicator. Mean BM loss in the present study was considerably lower (approximately 1.4\%) than that reported in other ultra-endurance studies of around 3-5\% [28-29,34]. As BM loss was not associated with $\mathrm{U}_{\mathrm{sg}}$ values in either sample it is likely that there is considerable error associated with the percentage BM loss data in this study. Firstly, it is important to establish euhydrated BM in participants in order to use BM change as an indicator of dehydration; secondly, over prolonged exercise substrate oxidation may contribute to changes in BM; for example, fat oxidation actually results in a net gain in BM as the mass of the carbon dioxide generated is less than the mass of the oxygen consumed [35-36]. The latter, however, is unlikely to result in profound changes and will result in similar errors in the other studies mentioned. It is likely that the main reason BM losses in the current study were less than those previously reported is that the participants in this study were weighed with their clothes on, which were likely heavier at the end due to wet weather conditions during both events.

\subsection{Hydration Status with Performance}

In the $\mathrm{LH}$ there was no association with $\mathrm{U}_{\mathrm{sg}}$ values and overall performance; however, in the OMM day one $U_{s g}$ values were positively associated with overall performance (Fig.1). This finding supports those of Clark et al. [4]; who reported that dehydration at the end of day two was positively associated with performance and Kao et al. [12]; who showed a significant relationship between percentage BM loss in a 24 hour ultra-marathon and performance. These findings contradict those of McConell et al. [10] and Fallowfield et al. [11], who previously reported negative effects of dehydration on endurance exercise performance in temperate climes. These conflicting results suggest that the relationship between hydration status and performance is complex and is not only likely due to temperature, exercise intensity, duration, and protocol, but also individual fitness and the physiological characteristics of the study participants. The different findings between the OMM and the LH could be due to the type of athletes the events attract. Whilst the LH is a smaller event comprising predominantly of local recreational athletes, the OMM is an international event attracting more experienced athletes. It has been proposed that top-level athletes may be better able to sustain performance, despite becoming dehydrated and it may be that the improvement in performance observed is due to them carrying less weight as fluid [4].

The possible physiological mechanisms for an increase in performance with dehydration are unclear, however the author hypothesises that dehydrated blood would lead to elevated blood glucose concentrations, which could result in increased transport of glucose into cells. In addition, blood concentration of haematocrit would also increase consequently enhancing blood oxygen levels. As glucose and oxygen are rate limiting in exercise performance, higher 
concentrations of these could be the reason for the improvement in performance seen.

This is therefore another study that challenges the well-established belief that euhydration is necessary to maintain performance during endurance exercise. Marino et al. [37] also did this when they reported participants performed as well when exercising for 60 minutes at $20^{\circ} \mathrm{C}$ when fully hydrated, as at $33^{\circ} \mathrm{C}$ without hydration. They investigated neuromuscular responses to hydration status and found adjustments were made allowing the attainment of similar core body temperatures, heart rates and performance times. It has been suggested [38] that these adjustments could be made as the exercise was self-paced, not of fixed-intensity like many of the exercise protocols used [10-11], which have led to the current recommendations. Whereas fixed intensity exercise to exhaustion only allows an all-or-nothing response, self-paced exercise of a set distance, like that performed in mountain marathons, allows continual behavioural adjustments that may affect performance [38].

Another theory is rather than dehydration itself negatively impacting on performance, it may be that not drinking to the satisfaction of thirst is responsible for the decrement in exercise performance found in many studies [39]. A meta-analysis of two research articles and eight individual studies reported drinking according to the dictate of thirst was associated with an increase in TT performance compared with a rate of drinking below $(+5.2 \pm 4.6 \%, p=$ $0.01)$, or above $(+2.4 \pm 5.0 \%, p=0.40)$, thirst [39]. This could account for why dehydration has not been found to negatively affect performance in many observational studies on "real-life" events when participants are free to drink as they desire [4,12], providing fluids are available.

Therefore, whilst laboratory-based studies are useful to determine physiological responses to exercise, field-based research is crucial to determine the effects in 'real-life' competition. Whilst this present study cannot conclude that dehydration improves performance, it suggests that moderate levels of dehydration in cool climates do not significantly reduce overall performance.

\subsection{Hydration Status and Cognitive Performance}

A greater decline was seen in performance in the reaction time tests in the LH sample than the OMM (Table 4). This is likely to be due to the continuous nature of the $\mathrm{LH}$ event allowing no time for rest and recovery. Improvements in performance were seen in the mean post-event scores of OMM competitors compared to mid-event scores, suggesting that the previously discussed learning effect associated with such tests outweighed any effects of fatigue. This indicates that perhaps further familiarisation is required with such tests before their use in the main study.

No significant differences were observed in either event between hydration status and cognitive performance in the CRT test (Fig.2). These findings were surprising; as it might be expected that dehydration would impair cognitive performance as adequate hydration is required for homeostasis and maintaining brain function [13]. Whilst the effects of dehydration on cognitive performance and brain function are not entirely known, there are several hormonal and cellular theories [40]. Dehydration leads to the activation of the hypothalamic-pituitary-adrenocortical axis and to increased production of the stress hormones i.e. cortisol. Studies have shown that hyercortisolaemia tends to worsen various cognitive functions such as perception, spatial ability and memory [41-42]. Other work has shown that changes in electrolyte concentrations during dehydration can alter brain activity [40]. However, it is likely that the reason no negative effect of dehydration on cognitive performance was seen in the present study, was that dehydration was not severe enough among these participants. A recent review of the literature has reported that the performance of simple attention tasks, such as reaction time, is not usually impaired at dehydration levels of $1-2 \%$ in cold environmental conditions [43]. There are however some studies that have found a deterioration, for example; a negative effect of dehydration on cognitive processing time has been reported in hill-walkers [14]. It is therefore possible that individuals respond differently to dehydration and some are better able to cope than others. The large range of results displayed in Figure 2 support this hypothesis and consequently a larger sample size would be needed to make any firm conclusions. Another possible factor in the response to dehydration may be age. In the hill-walking study [14] where dehydration did affect cognition, the mean age of the sample was $56 \pm 3$ years, older than the present study sample.

\subsection{Hydration Status and Well-Being}

Whilst the findings from this study suggest that the level of dehydration reported here does not significantly affect measures of performance, it does appear to have an impact on the athletes' well-being. In the OMM, RPE at the end of day one was associated with higher changes in $U_{\mathrm{sg}}$ values, which agrees with a laboratory-based study that reported an increased rating of fatigue with $2.8 \%$ dehydration versus euhydration [44]. Moran et al. [45] showed that perception of effort, using the Borg scale, was closely related to level of dehydration. They reported that an exercise intensity rated at $13.4 \pm 0.5$ ("somewhat hard") when participants were dehydrated to $1.1 \%$ of BM was rated at $17.6 \pm 0.3$ ("very hard") when dehydration was at $4.2 \%$ of BM. These findings suggest an association between the development of dehydration and subjective sensation of effort, which could ultimately impact on performance and also enjoyment of the event, the latter perhaps of particular importance to recreational athletes.

Incidence of headaches, cramps, stomach discomfort and nausea was also associated with higher $\mathrm{U}_{\mathrm{sg}}$ values. It is not surprising that those who were more dehydrated tended to suffer from headaches as this is a common symptom associated with dehydration and has been regularly reported by others [46]. Cramps were reported by a considerable proportion of subjects in both events $(44 \%$ in the OMM and 
$35 \%$ in the LH), again this is as expected as cramps have been cited by many as one of the most common complaints, particularly during endurance events such as triathlons and marathons, to be reported to medical staff $[1,47]$. Whilst dehydration alone may not be the sole cause of muscle cramps, studies have shown that remaining well hydrated could help delay the onset [48]. The findings that stomach discomfort and nausea were more common in those dehydrated is supported by the positive association observed in both studies between self-reported ratings of nausea and $U_{\text {sg }}$ values. However, athletes should be careful not to drink large volumes of fluid during exercise as that too can cause gastrointestinal discomfort [49]. The temperature of fluids should also be taken into account and may have varied greatly in this study; cold water from natural sources in comparison to warmer fluids carried in backpacks could have impacted upon levels consumed and symptoms experienced. This area warrants further investigation.

\subsection{Limitations}

It is evident from this study and from comparison with existing research that the effects of hydration status on exercise are complex. There is not only likely to be considerable individual variation, but the exercise protocols used, the level of dehydration, the environmental conditions and the method used to determine hydration status will all influence the outcome. As a result, the findings must take into account these factors and the limitations of the study.

Several limitations have been discussed such as the errors associated with using BM loss as a hydration marker, but it is important to note that urine values such as $U_{\mathrm{sg}}$ can also provide misleading results in certain cases. Levels of dehydration may have been under-reported in this study as ideally $U_{s g}$ values should be taken after several hours of stable hydration status. If a dehydrated individual consumes a substantial volume of hypotonic fluid prior to testing they will have large amounts of urine production long before euhydration is re-established and $\mathrm{U}_{\mathrm{sg}}$ values may indicate euhydration when the individual is still dehydrated [19]. It is also best practise to establish both $\mathrm{BM}$ and $\mathrm{U}_{\mathrm{sg}}$, and the relationship between them, in euhydrated participants before exercise commences so that individual results can be interpreted more accurately. As access to participants in a controlled environment, prior to the events, was not possible this could not be done and as a result the findings must be taken with caution.

The type and timing of fluid ingestion was not investigated which can significantly affect the ability of an individual to remain hydrated or to effectively re-hydrate. Several authors have written about the importance of electrolyte consumption during exercise. Coyle [2] stated that sodium should be included in fluids consumed during exercise lasting more than two hours, and Shirreffs and Sawka [50] explained that if rapid recovery is desired, such as during mid-camp events like the OMM, aggressive drinking of fluids and consumption of electrolytes is necessary to facilitate recovery for subsequent competition. Further research is therefore needed in this area, which takes into account not only quantity, but also quality of fluid consumption, in order to investigate whether mountain marathon athletes are currently meeting such recommendations.

\section{Conclusions}

A wide range of hydration levels were observed in these athletes; with some remaining well-hydrated, others finishing significantly dehydrated and others perhaps over drinking. Dehydration was not negatively associated with performance; however it was related to an increased incidence of various well-being measures such as headaches, cramps and nausea. Whilst the majority of elite athletes are primarily concerned with performance measures, recreational athletes are likely to be as concerned with factors that affect their enjoyment and so hydration strategies that could help to improve this are likely to be well-received.

Due to the wide variation in hydration levels observed it is likely that athletes would benefit from more individualised hydration strategies rather than blanket recommendations. Whilst it is difficult for recreational athletes to get individualised advice, it is possible to provide simple suggestions for athletes participating in particular events that take into account the nature and the environment of the event Athletes competing in the OMM should be made aware of situations where availability of water from natural sources may be limiting and that greater effort should be put into rehydration at mid-camp where water is available. To ensure less experienced athletes, who may be less in touch with their own needs, do not significantly over or under consume simple techniques such as monitoring urine frequency, volume and colour should be advised.

\section{References}

[1] D.J. Casa, P.M. Clarkson, and W.O. Roberts, "American College of Sports Medicine Roundtable on Hydration and Physical Activity: Consensus Statements." Current Sports Medicine Reports, vol. 4, pp.115-127, 2005.

[2] E.F. Coyle, "Fluid and fuel intake during exercise." Journal of Sports Sciences, vol. 22, pp.39-55, 2004.

[3] M.N. Sawka, "Physiological consequences of hypohydration: exercise performance and thermoregulation." Medicine and Science in Sports and Exercise, vol. 24, pp.657-670, 1992.

[4] H.R. Clark, M.E. Barker, and B,M. Corfe, "Nutritional Strategies of Mountain Marathon Competitors - An Observational Study." International Journal of Sport Nutrition and Exercise Metabolism, vol. 15, pp. 160-172, 2005.

[5] T.D. Noakes, Lore of Running. Champaign, IL: Human Kinetics, 2003.

[6] American College of Sports Medicine (ACSM), "Position Statement of the American College of Sports Medicine: prevention of heat injuries during distance running." Medicine and Science in Sports and Exercise, vol. 7, pp. vii-ix, 1975. 
[7] T.D. Hew, J.N. Chorley, J.C. Cianca, and J.G. Divine, "The incidence, risk factors, and clinical manifestations of hyponatremia in marathon runners." Clinical Journal of Sports medicine, vol. 13, pp.41-47, 2003.

[8] International Olympic Committee (IOC). "IOC consensus statement on sports nutrition 2010." Journal of Sports Sciences, vol. 29:sup1, pp. S3-S4, 2011.

[9] B. Murray, "Hydration and physical performance." Journal of the American College of Nutrition, vol. 26, pp. S542-S548, 2007.

[10] G.K. McConnell, C.M. Burge, S.L. Skinner, and M. Hargreaves, "Influence of ingested fluid volumes on physiological responses during prolonged exercise." Acta Physiologica Scandinavica, vol. 160, pp.149-156, 1997.

[11] J.L. Fallowfield, C. Williams, J. Booth, B.H. Choo, and S. Growns, "Effect of water ingestion on endurance capacity during prolonged running." Journal of Sports Sciences, vol. 14, pp.497-502, 1996.

[12] W.F. Kao, C.L. Shyu, and X.W. Yang, "Athletic performance and serial weight changes during 12- and 24-hour ultra-marathons." Clinical Journal of Sports Medicine, vol.18, pp.155-158, 2008.

[13] H.R. Lieberman, "Hydration and Cognition: A critical review and recommendations for future research." Journal of the American College of Nutrition, vol.26, pp.555S-561S, 2007.

[14] P.N. Ainslie, I.T. Campbell, K.N. Frayn, S.M. Humphreys, D.P.M. MacLaren, T. Reilly, and K.R. Weserterp, "Energy balance, metabolism, hydration and performance during strenuous hill walking: the effect of age." Journal of Applied Physiology, vol.93, pp.714-723, 2002.

[15] K.J. Ellis, "Selected body composition methods can be used in field studies." Journal of Nutrition, vol.131, pp.1589S-1595S, 2001.

[16] M. Stroud, "The nutritional demands of very prolonged exercise in man." Proceedings of the Nutrition Society, vol.57, pp.55-61, 1998.

[17] L.E. Armstrong, "Hydration Assessment Techniques." Nutrition Reviews, vol.63, pp.S40-S54, 2005.

[18] S.M. Shirreffs, "Markers of hydration status." European Journal of Clinical Nutrition, vol.57, pp.S6-S9, 2003.

[19] American College of Sports Medicine, "Position stand: Exercise and fluid replacement." Medicine and Science in Sports and Exercise, DOI: 10.1249/mss.0b013e31802ca.597, 2007.

[20] L.E. Armstrong, J.A. Soto, F.T. Hacker, J.R. Casa, S.A. Kavouras, and C.M. Maresh, "Urinary indices during dehydration, exercise and rehydration." International Journal of Sports Nutrition, vol.8, pp.345-355, 1998.

[21] M. Collardeau, J. Brisswalter, F. Vercruyssen, M. Audiffren, and C. Gaubault, "Single and Choice Reaction Time during Prolonged Exercise in Trained Subjects: Influence of Carbohydrate Availability." European Journal of Applied Physiology, vol.86, pp.150-156, 2001.

[22] A.J. Hill, and J.E. Blundell, "Sensitivity of appetite control system in obese subjects to nutritional and serotoninergic challenges." International Journal of Obesity, vol.14, pp.219-233, 1990 .
[23] P.N. Ainslie, I.T. Campbell, K.N. Frayn, S.M. Humphreys, D.P.M. MacLaren, T. Reilly, and K.R. Westerterp, "Energy balance, metabolism, hydration and performance during strenuous hill walking: the effect of age." Journal of Applied Physiology, vol.93, pp.714-723, 2002.

[24] G. Borg, "Perceived Exertion as an indicator of somatic stress." Scandinavian Journal of Rehabilitation Medicine, vol.2, pp.92-98, 1970.

[25] E. Blomstrand, P. Hassmen, B. Ekblom, and E.A. Newsholme, "Influence of ingesting a solution of branched-chain amino acids on perceived exertion during exercise." Acta Physiologica Scandinavica, vol.159, pp. 41-49, 1997.

[26] P.N. Ainslie, K. Abbas, I.T. Campbell, K.N. Frayn, M. Harvie, M.A. Keegan, D.P.M. MacLaren, I.A. MacDonald, K. Paramesh, and T. Reilly, "Metabolic and appetite responses to prolonged walking under three isoenergetic diets." Journal of Applied Physiology, vol.92, pp.2061-2070, 2002.

[27] A.C. Utter, J.I.E. Kang, R.J. Robertson, D.C. Nieman, E.C. Chaloupka, R.R. Suminski, and C.R. Piccinni, "Effect of carbohydrate ingestion on perceived exertion during a marathon." Medicine and Science in Sports and Exercise, vol.34, pp.1779-1784, 2002

[28] M. Kruesman, S. Bucher, M. Bovard, B. Kayser, and p.A. Bovier, "Nutrient intake and performance during a mountain marathon: an observational study." European Journal of Applied Physiology, vol. 94, pp. 151-157, 2005.

[29] N. Tam, T. Hew-Butler, E. Papadopoulou, H. Nolte, and T.D. Noakes, "Fluid intake and changes in blood biochemistry, running speed and body mass during an $80 \mathrm{~km}$ mountain trail race.” Medicina Sportiva, vol.13, pp.108-115, 2009.

[30] N.E. Kimber, J.J. Ross, S.L. Mason, and D.B. Speedy, "Energy balance during an ironman triathlon in male and female triathletes." International Journal of Sport Nutrition and Exercise Metabolism, vol. 12, pp. 47-62, 2002.

[31] G.W. Mack, C.A. Weseman, G.W. Langhans, H. Scherzer, C.M. Gillan, and E.R. Nadel, "Body fluid balance in dehydrated healthy older men: thirst and renal osmoregulation." Journal of Applied Physiology, vol.76, pp.1615-1623, 1994.

[32] P.A. Phillips, B.J. Rolls, J.G. Ledingham, and J.J. Morton, "Body fluid changes, thirst and drinking in man during free access to water." Physiology and Behaviour, vol.33, pp.357-363, 1984.

[33] W.L. Kenney, and P. Chiu, P. "Influence of age on thirst and fluid intake." Medicine and Science in Sports and Exercise, vol.33, pp.1524-1532, 2001.

[34] K.E. Fallon, E. Broad, M.W. Thompson, and P.A. Reull, "Nutritional and fluid intake in a $100-\mathrm{km}$ ultramarathon." International Journal of Sports Nutrition, vol. 8, pp. 24-35, 1998.

[35] R.J. Maughan, S.M. Shirreffs, and J.B. Leiper, "Errors in the estimation of hydration status from changes in body mass." Journal of Sports Sciences, vol.25, pp.797-804, 2007.

[36] R.F.G.J. King, C. Cooke, S. Carroll, and J. O'Hara, "Estimating changes in hydration status from changes in body mass: Considerations regarding metabolic water and glycogen storage." Journal of Sports Sciences, vol.26, pp. $1361-1363,2008$ 
[37] F.E. Marino, J. Kannon, and D. Kay, "Neuromuscular responses to hydration in moderate to warm ambient conditions during self-paced high-intensity exercise." British Journal of Sports Medicine, vol.44, pp.961-967, 2010.

[38] T. Mundel, "To drink or not to drink? Explaining "contradictory findings" in fluid replacement and exercise performance: evidence from a more valid model for real-life competition." British Journal of Sports Medicine, vol.45, pp.1-2, 2011.

[39] E.D.B. Goulet, "Effect of exercise-induced dehydration on time-trial exercise performance: a meta-analysis." British Journal of Sports Medicine, vol.45, pp.1149-1156, 2011.

[40] M.MG. Wilson, and J.E. Morley, "Impaired cognitive function and mental performance in mild dehydration." European Journal of Clinical Nutrition, vol.57, pp.S24-S29, 2003.

[41] K.E. D'Anci, F. Constant, and I.H. Rosenberg, I.H. "Hydration and cognitive function in children." Nutrition Reviews, vol.64, pp.457-464, 2006.

[42] H.R. Lieberman, G.P. Bathalon, C.M. Falco, F.M. Kramer, C.A. Morgan, and P. Niro, "Severe decrements in cognitive functions and mood induced by sleep loss, heat, dehydration, and undernutrition during simulated combat." Biological Psychiatry, vol.57, pp.422-429, 2005.

[43] A. Adan, "Cognitive performance and dehydration." Journal of the American College of Nutrition, vol.31, pp.71-78, 2012.

[44] C. Cian, N. Koulmann, P. Barraud, C. Raphel, C. Jimenez, and B. Melin, "Influence of variation in body hydration on cognitive function: effect of hyperhydration, heat stress, and exercise-induced dehydration.” Journal of Psychophysiology, vol.14, pp.29-36, 2000.

[45] D.S. Moran, S.J. Montain, and K.B. Pandolf, "Evaluation of different levels of hydration using a new physiological strain index." American Journal of Physiology: Regulatory, Integrative and Comparative Physiology, vol.275, pp.R854-R860, 1998.

[46] R.J. Maughan, "Impact of mild dehydration on wellness and on exercise performance." European Journal of Clinical Nutrition, vol.57, pp.S19-S23, 2003.

[47] M.P. Schwellnus, "Muscle Cramping in the Marathon: Aetiology and Risk Factors." Sports Medicine, vol.37, pp.364-367, 2007.

[48] A.P. Jung, P.A. Bishop, A. Al-Nawwas, and B. Dale, "Influence of hydration and electrolyte supplementation on incidence and time to onset of exercise-associated muscle cramps." Journal of Athletic Training, vol.40, pp.71-75, 2005.

[49] T.A. Robinson, J.A. Hawley, G.S. Palmer, G.R. Wilson, D.A. Gray, and T.D. Noakes, "Water ingestion does not improve 1-h cycling performance in moderate ambient temperatures." European Journal of Applied Physiology and Occupational Physiology, vol.71, pp.153-160, 1995.

[50] S.M. Shirreffs, and M.N. Sawka, "Fluid and electrolyte needs for training, competition and recovery." Journal of Sports Sciences, vol.29, pp.S39-S46, 2011. 